\title{
Caracterización diagnóstica de la consulta externa de nefrología en el Hospital Nacional Dos de Mayo, 2011-2016
}

\section{A descriptive characterization of diagnoses made in the nephrology outpatient clinic in Hospital Nacional Dos de Mayo, 2011-2016}

Correspondencia

Luis Arellan Bravo

luisarellan@gmail.com

Recibido: 28/03/2018

Aprobado: 11/04/2018

Citar como: Valdez-Yánez $G$, Arellán-Bravo L, HernándezPacheco J, Velásquez-Castillo P, Matias-Tasayco V.

Caracterización diagnóstica de la consulta externa de nefrología en el Hospital Nacional Dos de Mayo, 2011-2016. Acta Med Peru. 2018;35(2):138-9

\author{
Guillermo Valdez-Yánez ${ }^{1,2, a}$, Luis Arellán-Bravo ${ }^{1, b}$, Javier Hernández- \\ Pacheco ${ }^{1, a}$, Percy Velásquez-Castillo ${ }^{1, b}$, Vanessa Matias-Tasayco ${ }^{1, b}$ \\ 1 Servicio de Nefrología, Hospital Nacional Dos de Mayo Lima, Perú. \\ 2 Departamento de Medicina Interna, Universidad Nacional Mayor de San Marcos. Lima, Perú. \\ a Medico nefrólogo; b Médico residente de Nefrología
}

\section{Sr. Editor:}

El Hospital Nacional Dos de Mayo (HN2M) es considerado un centro de referencia nacional para el manejo de problemas renales que atiende alrededor de 5000 consultas externas anuales en el servicio de nefrología ${ }^{[1,2]}$. Actualmente, la enfermedad renal crónica (ERC) es un problema de salud pública a nivel mundial. Un estudio realizado en Lima y Tumbes estima que la prevalencia de enfermedad renal crónica (ERC) es de $16,8 \%{ }^{[3]}$. Asimismo, se reporta que la prevalencia de ERC en la seguridad social (EsSalud) fue del $18 \%{ }^{[4]}$. Estas cifras ponen de manifiesto la importancia de brindar un diagnóstico y tratamiento temprano para evitar el impacto y consecuencias de la enfermedad.

En tal sentido, realizamos un análisis descriptivo de los diagnósticos según CIE-10 reportados en las fichas de registro del sistema de información HIS en los últimos seis años por los servicios de consulta externa de nefrología del HN2M. Los resultados se muestran en la Tabla 1.

Tabla 1. Distribución de los diagnósticos según CIE-10 reportados en las fichas de registro del sistema de información HIS, 2011-2016.

\begin{tabular}{lccc}
\multicolumn{1}{c}{ Diagnóstico (CIE-10) } & Total (\%) & Mujer & Hombre \\
\hline Enfermedad renal crónica terminal (N18.0) & $5716(19,7)$ & 2781 & 2935 \\
Síndrome nefrítico (N03.9) & $1422(4,9)$ & 718 & 705 \\
Diabetes mellitus tipo 2 (E11.X) & $945(3,2)$ & 606 & 340 \\
Hipertensión arterial (I10.X) & $942(3,2)$ & 624 & 318 \\
Síndrome nefrótico (N04.9) & $264(0,9)$ & 65 & 199 \\
Otros diagnósticos & $19716(68,1)$ & 10844 & 8872 \\
\hline
\end{tabular}

Durante el periodo de estudio se atendieron un total de 15638 (53,9\%) mujeres y 13369 (46,1\%) hombres, siendo la causa más frecuente de consulta externa fue la enfermedad renal crónica terminal $(19,7 \%)$. Esto pone de manifiesto el alto número de pacientes que son atendidos en estadios finales de la enfermedad renal, posiblemente por una referencia tardía al nefrólogo o baja disponibilidad de servicios de nefrología en el país. 
Al respecto, en los hospitales de Lima se encuentra que el $45 \%$ de los pacientes diabéticos atendidos en el servicio de nefrología fueron referidos con síntomas de nefropatía, y el inicio de los síntomas fue menor a seis meses en el $64,4 \%$ de los pacientes ${ }^{[5]}$. Por ello, es importante que se refieran oportunamente a los pacientes a los servicios de nefrología y así evitar su manejo tardío.

De esta manera, hacemos énfasis en la importancia de reportar las estadísticas hospitalarias de las unidades de nefrología, además de mostrar las principales patologías renales que son atendidas en un hospital de referencia nacional.

\title{
REFERENCIAS BIBLIOGRÁFICAS
}

1. Salaverry O, Cárdenas-Rojas D. Establecimientos asistenciales de sector salud, Perú 2009: (PDF). Rev Peru Med Exp Salud Publica. 2009;26(2):264-7.

2. Hospital Dos de Mayo. Historia del Hospital Dos de Mayo [Internet]. Lima: c2017; [citado el 4 de abril de 2017]. Disponible en: http:// hdosdemayo.gob.pe/portal/resena-historica/

3. Francis ER, Kuo CC, Bernabe-Ortiz A, Nessel L, Gilman RH, Checkley $\mathrm{W}$, Miranda JJ, et al. Burden of chronic kidney disease in resource- limited settings from Peru: a population-based study. BMC Nephrol. 2015;16:114.

4. Herrera-Añazco P, Taype-Rondan A, Lazo-Porras M, Alberto Quintanilla E, Ortiz-Soriano VM, Hernandez AV. Prevalence of chronic kidney disease in Peruvian primary care setting. BMC Nephrol. 2017;18:246.

5. Herrera Añazco $P$, Bonilla Vargas L, Palacios Guillén $M$, Valencia Rodríguez J, Sánchez Riva F, Salomé Luna J, et al. Características clínicas de los pacientes diabéticos que acuden por primera vez a una consulta nefrológica en hospitales públicos de Lima. An Fac med. 2014;75(1):25-9.

\section{Ahora puede enviar sus artículos para Acta Médica Peruana en nuestro Open Journal System:}

\author{
www.amp.cmp.org.pe
}

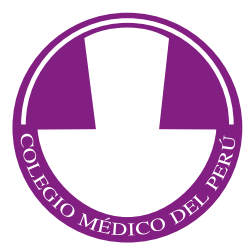

